\title{
On Some Structural Importance of System Components
}

\author{
Fan C. Meng \\ Academia Sinica
}

\begin{abstract}
In this note a new method of comparing component structural importance is introduced and compared to other existing ones. Especially, relationships of the new comparison method to the H-importance due to Hwang $(2001,2005)$, the criticality ordering due to Boland et al. (1989) and Birnbaum importance are obtained. Illustrative examples are given.
\end{abstract}

Key words: Binary coherent system, Birnbaum importance measure, minimal path (cut) set, path (cut) set.

\section{Introduction}

Consider a binary coherent system $(C, \phi)$ composed of $n$ independent components, where $C=\{1,2, \ldots, n\}$ denotes the index set of the $n$ components, and $\phi$, a mapping from $\{0,1\}^{n}$ to $\{0,1\}$, denotes the (nondecreasing) structure function of the system. Denote by $p_{i}$ the reliability of the $i$ th component, and by $h(\underline{\mathrm{p}})=h\left(p_{1}, \ldots, p_{n}\right)$ the system reliability function. A vector $\mathrm{x}$ for which $\phi(\underline{x})=0$ (1) is called a cut (path) vector. A minimal cut (path) vector is a cut (path) vector $\mathrm{x}$, and $\phi(\mathrm{y})=1(0)$ for all $\mathrm{y}>(<) \mathrm{x}$, where $\mathrm{y}>\underline{\mathrm{x}}$ means $\mathrm{y}_{i} \geq x_{i}$ for all $i$ and $\mathrm{y}_{i}>x_{i}$ for some $i$. For any cut (path) vector $\mathrm{x}$ the index subset $\left\{i: x_{i}=0\right\}\left(\left\{i: x_{i}=1\right\}\right)$ is the corresponding cut (path) set. If $\mathrm{x}$ is a minimal cut (path) vector then $\left\{i: x_{i}=0\right\}\left(\left\{i: x_{i}=1\right\}\right)$ is called a minimal cut (path) set for the system. See Barlow and Proschan (1981) for a full account of the theory of binary coherent systems.

Various measures (orderings) of component importance for binary coherent systems have been introduced in the literature. The most fundamental one is Birnbaum reliability importance measure, defined by

$$
I_{h}(i ; \underline{\mathrm{p}})=h\left(1_{i}, \underline{\mathrm{p}}\right)-h\left(0_{i}, \underline{\mathrm{p}}\right)=\partial h(\underline{\mathrm{p}}) / \partial p_{i},
$$

where $\left({ }_{i}, \underline{\mathrm{p}}\right)=\left(p_{1}, \cdots, p_{i-1}, \cdot_{i}, p_{i+1}, \cdots, p_{n}\right)$.

The Birnbaum reliability importance of components may be used to evaluate the effect of an improvement in component reliability on system reliability (see 
Barlow and Proschan (1981)). In the case where $p_{1}=\cdots=p_{n}=1 / 2$, the Birnbaum reliability importance measure reduces to Birnbaum structural importance measure, denoted by $I_{\phi}(i)$,

$$
I_{\phi}(i)=I_{h}(i ; 1 / 2, \cdots, 1 / 2)=\frac{1}{2^{n-1}}\left|\underline{\mathrm{x}}: \phi\left(1_{i}, \underline{\mathrm{x}}\right)>\phi\left(0_{i}, \underline{\mathrm{x}}\right)\right|,
$$

where $\mathrm{x} \in\{0,1\}^{n-1}$, and $|\cdot|$ denotes set cardinality.

In the absence of information about component reliabilities, the Birnbaum structural importance measure provides a fair comparison of relative importance among system components. However, for systems with highly reliable components, using the Birnbaum structural importance may give a misleading picture of which components are most important. Butler (1979) developed an alternative structural ordering, namely the cut-importance ordering, and obtained a relationship between the cut-importance and the Birnbaum reliability importance measure for high values of $\mathrm{p}$. Since structural importance of a component represents importance of the node (position), we use the terms structural importance of component $i$ or importance of node $i$ alternatively in this note without ambiguity.

Definition 1.1: (Butler (1979)) For each component $s$, let $d_{i j}^{(s)}$ denote the number of unions of $i$ distinct minimal cut sets such that the union contains exactly $j$ nodes and includes node $s$. Let $b_{j}^{(s)}=\sum_{i \geq 1}(-1)^{i-1} d_{i j}^{(s)}$, and let $\underline{\mathrm{b}}^{(s)}=\left(b_{1}^{(s)}, \ldots, b_{n}^{(s)}\right)$. Node $s$ is said to be more cut-important than node $t$, denoted by $s>_{c} t$, if and only if $\underline{\mathrm{b}}^{(s)} \succ \underline{\mathrm{b}}^{(t)}$, where $\succ$ denotes lexicographic ordering. Nodes $s$ and $t$ are equally cut-important, denoted by $s={ }_{c} t$, if and only if $\underline{\underline{b}}^{(s)}=\underline{\mathrm{b}}^{(t)}$.

Theorem 1.2: (Butler (1979)) For $\mathrm{p}=(p, p, \cdots, p)$ and the scalar $p$ is sufficiently close to one, the cut-importance ordering $>_{c}$ and the ordering induced by $I_{h}(i ; \underline{\mathrm{p}})$ are identical.

Later, Boland et al. (1989) introduced the following notion of criticality. Based on the structural criticality ordering, they developed a principle of pairwise rearrangement of components to improve system reliability.

Definition 1.3: Component $i$ is said to be more critical than component $j$ for $\phi$, denoted by $i>^{c} j$, if $\phi\left(1_{i}, 0_{j}, \underline{\mathrm{x}}\right) \geq \phi\left(0_{i}, 1_{j}, \underline{\mathrm{x}}\right)$ for all $\underline{\mathrm{x}} \in\{0,1\}^{n-2}$, and strict inequality holds for some $\underline{x}$. If equality holds for all $\underline{x}$, components $i$ and $j$ are said to be permutation equivalent, denoted by $i={ }^{c} j$.

It is shown in Boland et al. (1989) that the criticality ordering is in some sense stronger than that of the Birnbaum structural importance: 
Theorem 1.4: (Boland et al. (1989)) $i>^{c} j \Longrightarrow I_{\phi}(i)>I_{\phi}(j)$.

The relationships of the structural criticality and the cut-importance to the Birnbaum reliability importance are presented in Meng (1995):

(I) $i>^{c} j \Longleftrightarrow I_{h}\left(i ; \alpha_{j}, \underline{\mathrm{p}}\right)>I_{h}\left(j ; \alpha_{i}, \underline{\mathrm{p}}\right)$ for all $\underline{\mathrm{p}} \in(0,1)^{n-2}$, all $\alpha \in[0,1]$;

(II) $i>_{c} j \Longleftrightarrow I_{h}\left(i ; \alpha_{j}, \underline{\mathrm{p}}\right)>I_{h}\left(j ; \alpha_{i}, \underline{\mathrm{p}}\right)$ for all $\mathbf{p}=(p, p, \cdots, p)$ where $p \rightarrow 1$, all $\alpha \in[0,1]$.

(III) It readily follows that $i>^{c} j \Longrightarrow i>_{c} j$, from (I) and (II).

Recently, Hwang $(2001,2005)$ introduced the following two new indices of component structural importance, based on the cut sets (path sets) and minimal cut sets of a binary coherent system.

Definition 1.5: Components $i$ is said to be more H-important than $j$ if $\left|C_{i}(d)\right| \geq$ $\left|C_{j}(d)\right|\left(\left|P_{i}(d)\right| \geq\left|P_{j}(d)\right|\right)$ for all $1 \leq d \leq n$, where $C_{i}(d)\left(P_{i}(d)\right)$ is the set of cut sets (path sets) of size $d$ that contains $i$.

Definition 1.6: Components $i$ is said to be more $\bar{H}$-important than $j$ if $\left|\bar{C}_{i}(d)\right| \geq$ $\left|\bar{C}_{j}(d)\right|$ for all $1 \leq d \leq n$, where $\bar{C}_{i}(d)$ is the set of minimal cut sets of size $d$ that contains $i$.

It is shown in Hwang $(2001,2005)$ that the notion of H- $(\bar{H})$-importance is between the criticality and the cut-importance in the sense that

(i) $i>^{c} j \Longrightarrow i$ is more $\mathrm{H}$-important than $j \Longrightarrow i>_{c} j$;

(ii) $i>^{c} j \Longrightarrow i$ is more $\bar{H}$-important than $j \Longrightarrow i>_{c} j$.

The above implications in (i) and (ii) are not reversible (see examples in section 3 of this note).

\section{Results}

First, we introduce a new method of comparing component relative importance, instead of using quantitative measures.

Definition 2.1: For $(i, j) \subset\{1,2, \cdots, n\}$ and $1 \leq d \leq n-1, I_{\phi ; d}(i) \geq I_{\phi ; d}(j)$ if

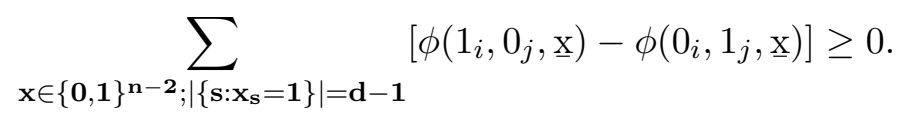

Remark. If $I_{\phi ; d}(i)>I_{\phi ; d}(j)$, then there exists at least an $\mathbf{x} \in\{0,1\}^{n-2}, \sum_{t \neq i, j} x_{t}=$ $d-1$, such that $\phi\left(1_{i}, 0_{j}, \underline{\mathrm{x}}\right)=1$ and $\phi\left(0_{i}, 1_{j}, \underline{\mathrm{x}}\right)=0$. That is, there exists a path set of size $d$ which contains $i$ but not $j$.

Lemma 2.2: The Birnbaum structural importance, $I_{\phi}(i) \geq I_{\phi}(j)$

$$
\Longleftrightarrow\left|\left\{\underline{\mathrm{x}}: \phi\left(1_{i}, 0_{j}, \underline{\mathrm{x}}\right)>\phi\left(0_{i}, 1_{j}, \underline{\mathrm{x}}\right)\right\}\right| \geq\left|\left\{\underline{\mathrm{x}}: \phi\left(1_{i}, 0_{j}, \underline{\mathrm{x}}\right)<\phi\left(0_{i}, 1_{j}, \underline{\mathrm{x}}\right)\right\}\right|
$$


$\left.\Longleftrightarrow \sum_{\mathbf{x} \in\{0,1\}^{n}}\left[\phi\left(1_{i}, 0_{j}, \underline{\mathrm{x}}\right)-\phi\left(0_{i}, 1_{j}, \underline{\mathrm{x}}\right)\right\}\right] \geq 0$.

\section{Proof:}

$$
\begin{aligned}
I_{\phi}(i)= & \frac{1}{2^{n-1}}\left|\left\{\underline{\mathrm{x}}: \phi\left(1_{i}, \underline{\mathrm{x}}\right)>\phi\left(0_{i}, \underline{\mathrm{x}}\right)\right\}\right| \\
= & \frac{1}{2^{n-1}}\left|\left\{\underline{\mathrm{z}}: \phi\left(1_{i}, 1_{j}, \underline{\mathrm{z}}\right)>\phi\left(0_{i}, 1_{j}, \underline{\mathrm{z}}\right)\right\}\right|+\frac{1}{2^{n-1}}\left|\left\{\underline{\mathrm{z}}: \phi\left(1_{i}, 0_{j}, \underline{\mathrm{z}}\right)>\phi\left(0_{i}, 0_{j}, \underline{\mathrm{z}}\right)\right\}\right| \\
= & \frac{1}{2^{n-1}}\left|\left\{\underline{\mathrm{z}}: \phi\left(1_{i}, 1_{j}, \underline{\mathrm{z}}\right)=1\right\}\right|-\frac{1}{2^{n-1}}\left|\left\{\underline{\mathrm{z}}: \phi\left(0_{i}, 1_{j}, \underline{\mathrm{z}}\right)=1\right\}\right| \\
& +\frac{1}{2^{n-1}}\left|\left\{\underline{\mathrm{z}}: \phi\left(1_{i}, 0_{j}, \underline{\mathrm{z}}\right)=1\right\}\right|-\frac{1}{2^{n-1}}\left|\left\{\underline{\mathrm{z}}: \phi\left(0_{i}, 0_{j}, \underline{\mathrm{z}}\right)=1\right\}\right| .
\end{aligned}
$$

From the above expression, we easily see that

$$
\begin{aligned}
& I_{\phi}(i)-I_{\phi}(j)=\frac{1}{2^{n-2}}\left|\left\{\underline{\mathrm{z}}: \phi\left(1_{i}, 0_{j}, \underline{\mathrm{z}}\right)=1\right\}\right|-\frac{1}{2^{n-2}}\left|\left\{\underline{\mathrm{z}}: \phi\left(0_{i}, 1_{j}, \underline{\mathrm{z}}\right)=1\right\}\right| \\
& \quad=\frac{1}{2^{n-2}}\left|\left\{\underline{\mathrm{z}}: \phi\left(1_{i}, 0_{j}, \underline{\mathrm{z}}\right)>\phi\left(0_{i}, 1_{j}, \underline{\mathrm{z}}\right)\right\}\right|-\frac{1}{2^{n-2}}\left|\left\{\underline{\mathrm{z}}: \phi\left(1_{i}, 0_{j}, \underline{\mathrm{x}}\right)<\phi\left(0_{i}, 1_{j}, \underline{\mathrm{z}}\right)\right\}\right| .
\end{aligned}
$$

Thus, $I_{\phi}(i) \geq I_{\phi}(j)$

$$
\begin{aligned}
& \Longleftrightarrow\left|\left\{\underline{\mathrm{x}}: \phi\left(1_{i}, 0_{j}, \underline{\mathrm{x}}\right)>\phi\left(0_{i}, 1_{j}, \underline{\mathrm{x}}\right)\right\}\right| \geq\left|\left\{\underline{\mathrm{x}}: \phi\left(1_{i}, 0_{j}, \underline{\mathrm{x}}\right)<\phi\left(0_{i}, 1_{j}, \underline{\mathrm{x}}\right)\right\}\right| \\
& \left.\Longleftrightarrow \sum_{\mathbf{x}}\left[\phi\left(1_{i}, 0_{j}, \underline{\mathrm{x}}\right)-\phi\left(0_{i}, 1_{j}, \underline{\mathrm{x}}\right)\right\}\right] \geq 0 .
\end{aligned}
$$

Next we show that the structural ordering in Definition 2.1 is essentially equivalent to the one induced by the number of cut sets (path sets) introduced in Hwang (2001).

Lemma 2.3: $\left|C_{i}(d)\right| \geq\left|C_{j}(d)\right| \Longleftrightarrow I_{\phi ; n-d}(i) \geq I_{\phi ; n-d}(j)(d=1, \cdots, n-1)$.

Proof: The sets $C_{i}(d)$ and $C_{j}(d)$ can be decomposed as unions of two disjoint sets. That is, $C_{i}(d)=C_{i j}(d) \cup C_{i \bar{j}}(d)$ and $C_{j}(d)=C_{i j}(d) \cup C_{\overline{i j}(d)}$, where $C_{i j}(d)$ denotes cut sets that contains both $i$ and $j$; and $C_{i \bar{j}}(d)\left(C_{\bar{i} j}(d)\right)$ denotes cut sets that contains $i(j)$ but not $j(i)$. Thus,

$$
\begin{aligned}
& \left|C_{i}(d)\right| \geq\left|C_{j}(d)\right| \Longleftrightarrow\left|C_{i \bar{j}}(d)\right| \geq\left|C_{\bar{i} j}(d)\right| \\
& \Longleftrightarrow\left|\left\{(i, A): A \subset\{1, \cdots, n\} \backslash\{i\} ;(i, A) \in C_{i \bar{j}}(d),|A|=d-1\right\}\right| \\
& \geq\left|\left\{(j, A): A \subset\{1, \cdots, n\} \backslash\{j\} ;(j, A) \in C_{\overline{i j}}(d),|A|=d-1\right\}\right| \\
& \Longleftrightarrow\left|\left\{\underline{\mathrm{x}}: \phi\left(1_{i}, 0_{j}, \underline{\mathrm{x}}\right)>\phi\left(0_{i}, 1_{j}, \underline{\mathrm{x}}\right) ; \sum_{k \neq i, j} x_{k}=n-d-1\right\}\right| \\
& \quad \geq\left|\left\{\underline{\mathrm{x}}: \phi\left(1_{i}, 0_{j}, \underline{\mathrm{x}}\right)<\phi\left(0_{i}, 1_{j}, \underline{\mathrm{x}}\right) ; \sum_{k \neq i, j} x_{k}=n-d-1\right\}\right| \\
& \quad \Longleftrightarrow \sum_{\mathbf{x} \in\{\mathbf{0}, \mathbf{1}\}^{\mathbf{n}-\mathbf{2}} ;\left|\left\{\mathbf{s}: \mathbf{x}_{\mathbf{s}}=\mathbf{1}\right\}\right|=\mathbf{n}-\mathbf{d}-\mathbf{1}}\left[\phi\left(1_{i}, 0_{j}, \underline{\mathbf{x}}\right)-\phi\left(0_{i}, 1_{j}, \underline{\mathbf{x}}\right)\right] \geq 0 .
\end{aligned}
$$

The conclusion follows.

The Birnbaum structure importance $I_{\phi}(i)$ is identical to the Birnbaum reliability importance $I_{h}(i ; \underline{p})$ evaluated at $\mathrm{p}=(1 / 2, \cdots, 1 / 2)$. It is shown in Hwang (2001) that if components i is more H-important than $j$, then $i$ dominates $j$ in the universal Birnbaum comparison, namely, $I_{h}(i ; \underline{\mathrm{p}}) \geq I_{h}(j ; \underline{\mathrm{p}})$ for all $\mathbf{p}=(p, \cdots, p)$, all $p \in(0,1)$. The following theorem slightly strengthens this result.

Theorem 2.4: If $I_{\phi ; d}(i) \geq I_{\phi ; d}(j)$ for all $1 \leq d \leq n-1$, then $I_{h}\left(i ; \alpha_{j}, \mathbf{p}\right) \geq I_{h}\left(j ; \alpha_{i}, \mathbf{p}\right)$ for all $\alpha \in[0,1]$, all $\mathbf{p}=(p, \cdots, p)$. 
Proof.

$$
\begin{aligned}
I_{h}(i ; \underline{\mathrm{p}})= & h\left(1_{i}, \underline{\mathrm{p}}\right)-h\left(0_{i}, \underline{\mathrm{p}}\right) \\
= & p_{j} h\left(1_{i}, 1_{j}, \underline{\mathrm{p}}\right)+\left(1-p_{j}\right) h\left(1_{i}, 0_{j}, \underline{\mathrm{p}}\right) \\
& -p_{j} h\left(0_{i}, 1_{j}, \underline{\mathrm{p}}\right)-\left(1-p_{j}\right) h\left(0_{i}, 0_{j}, \underline{\mathrm{p}}\right) .
\end{aligned}
$$

From the above expression, we see that

$$
\begin{aligned}
I_{h}(i ; \underline{\mathrm{p}})-I_{h}(j ; \underline{\mathrm{p}})= & \left(p_{j}-p_{i}\right) h\left(1_{i}, 1_{j}, \underline{\mathrm{p}}\right)+\left(1-p_{j}+p_{i}\right) h\left(1_{i}, 0_{j}, \underline{\mathrm{p}}\right) \\
& -\left(1-p_{i}+p_{j}\right) h\left(0_{i}, 1_{j}, \underline{\mathrm{p}}\right)+\left(p_{j}-p_{i}\right) h\left(0_{i}, 0_{j}, \underline{\mathrm{p}}\right)
\end{aligned}
$$

When $p_{i}=p_{j}=\alpha$ and $\underline{\mathrm{p}}=(p, \cdots, p)$, the above equality reduces to

$$
\begin{aligned}
& I_{h}\left(i, \alpha_{j} ; p\right)-I_{h}\left(j, \alpha_{i} ; p\right)=h\left(1_{i}, 0_{j}, p\right)-h\left(0_{i}, 1_{j}, p\right) \\
& \quad=E_{p}\left\{\phi\left(1_{i}, 0_{j}, \underline{\mathrm{X}}\right)=1\right\}-E_{p}\left\{\phi\left(0_{i}, 1_{j}, \mathrm{X}\right)=1\right\} \\
& \quad=E_{p}\left\{\phi\left(1_{i}, 0_{j}, \underline{\mathrm{X}}\right)-\phi\left(0_{i}, 1_{j}, \underline{\mathrm{X}}\right)\right\} \\
& =\sum_{d=0}^{n-2} p^{d}(1-p)^{n-d-2} \sum_{\mathbf{x}:\left|\left\{s ; x_{s}=1\right\}\right|=d}\left[\phi\left(1_{i}, 0_{j}, \underline{\mathrm{x}}\right)-\phi\left(0_{i}, 1_{j}, \underline{\mathrm{x}}\right)\right] \\
& \geq 0 \text { for all } p \text {, by assumption. }
\end{aligned}
$$

From Lemma 2.3, Theorem 2.4 and result (II) in section 1, we conclude that more H-important implies more cut-important. This fact was proved, but the proof in Hwang (2001) is rather complicated.

The main result obtained in Boland et al. (1989) provides a principle of rearrangement of components in order to achieve better system reliability. This principle can be rephrased as: $i>^{c} j$ if and only if $h\left(\alpha_{i}, \beta_{j} ; \mathrm{p}\right) \geq h\left(\beta_{i}, \alpha_{j} ; \mathrm{p}\right)$ for all $1 \geq \alpha>\beta \geq 0$, all $\underline{p}$, with strict inequality holding for some $\underline{1}>\underline{\mathrm{p}}>\underline{0}$. Under a weaker than criticality assumption on component importance, we obtain the following theorem pertaining to rearrangement of components.

Theorem 2.5: If $I_{\phi ; d}(i) \geq I_{\phi ; d}(j)$ for all $1 \leq d \leq n-1$, then $h\left(\alpha_{i}, \beta_{j} ; \mathbf{p}\right) \geq$ $h\left(\beta_{i}, \alpha_{j} ; \mathbf{p}\right)$ for all $1 \geq \alpha>\beta \geq 0$, all $\mathbf{p}=(p, \cdots, p)$.

\section{Proof:}

$$
\begin{aligned}
h\left(\alpha_{i}, \beta_{j}, \mathbf{p}\right)= & \alpha h\left(1_{i}, \beta_{j}, \underline{\mathrm{p}}\right)+(1-\alpha) h\left(0_{i}, \beta_{j}, \underline{\mathrm{p}}\right) \\
= & \alpha \beta h\left(1_{i}, 1_{j}, \underline{\mathrm{p}}\right)+\alpha(1-\beta) h\left(1_{i}, 0_{j}, \underline{\mathrm{p}}\right) \\
& +(1-\alpha) \beta h\left(0_{i}, 1_{j}, \underline{\mathrm{p}}\right)+(1-\alpha)(1-\beta) h\left(0_{i}, 0_{j}, \underline{\mathrm{p}}\right) .
\end{aligned}
$$

Thus,

$$
\begin{aligned}
h\left(\alpha_{i}, \beta_{j}, \mathbf{p}\right)-h\left(\beta_{i}, \alpha_{j}, \mathbf{p}\right)= & (\alpha-\beta)\left[h\left(1_{i}, 0_{j}, \mathbf{p}\right)-h\left(0_{i}, 1_{j}, \mathbf{p}\right)\right] \\
= & (\alpha-\beta)\left[I_{h}\left(i ; \alpha_{j}, \mathbf{p}\right)-I_{h}\left(j ; \alpha_{i}, \mathbf{p}\right)\right] \\
& \text { (see the proof of Theorem 2.4). }
\end{aligned}
$$

When $\mathbf{p}=(p, \cdots, p)$, from the proof of Theorem 2.4,

$$
h\left(1_{i}, 0_{j}, p\right)-h\left(0_{i}, 1_{j}, p\right)=I_{h}\left(i ; \alpha_{j}, p\right)-I_{h}\left(j ; \alpha_{i}, p\right) \geq 0 .
$$


Hence, $h\left(\alpha_{i}, \beta_{j}, \mathbf{p}\right) \geq h\left(\beta_{i}, \alpha_{j}, \mathbf{p}\right)$ holds for all $1 \geq \alpha>\beta \geq 0$, all $\mathbf{p}=(p, \cdots, p)$.

\section{Illustrative examples}

In this section examples are given to illustrate that the implication relationships obtained in Hwang $(2001,2005)$, concerning criticality, cut importance, H-importance and $\bar{H}$-importance, are not reversible.

Example 1: (from Butler (1979))

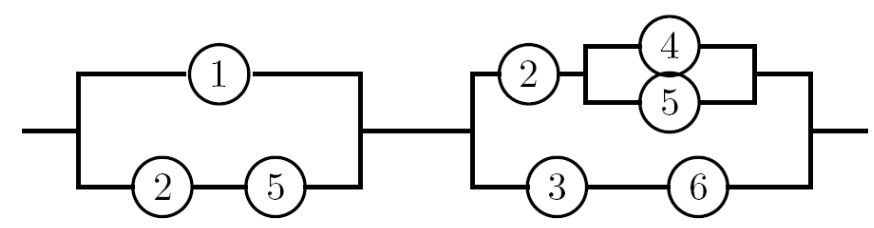

Figure 1: A six-component system

The structure function, $\phi(\underline{\mathrm{x}})=\left[1-\left(1-x_{1}\right)\left(1-x_{5}\right)\right]\left[1-\left(1-x_{2}\right)\left(1-x_{3}\right)\right]$.

$\left[1-\left(1-x_{2}\right)\left(1-x_{6}\right)\right]\left[\left(1-\left(1-x_{4}\right)\left(1-x_{5}\right)\left(1-x_{6}\right)\right]\right.$.

$\left[1-\left(1-x_{3}\right)\left(1-x_{4}\right)\left(1-x_{5}\right)\right]\left[1-\left(1-x_{1}\right)\left(1-x_{2}\right)\right]$.

The minimal cut sets are $\{1,5\},\{2,3\},\{2,6\},\{4,5,6\},\{3,4,5\}$ and $\{1,2\}$. It was shown in Butler (1979) that $2>_{c} 1>_{c} 5>_{c} 3={ }_{c} 6>_{c} 4$. It is easy to see that $\bar{C}_{6}(3)=1$, and $\bar{C}_{4}(3)=2$. Thus, component 6 is not more $\bar{H}$-important than component 4 . It is seen that $>_{c} \Longrightarrow$ more $\bar{H}$-important. Node 1 and node 2 are not comparable by their criticality, noting that $\phi(0,1,1,1,0,1)<\phi(1,0,1,1,0,1)$ and $\phi(1,0,0,1,1,1)<\phi(0,1,0,1,1,1)$. Also, $\bar{C}_{1}(2)=2$, and $C_{1}(3)=0 ; \bar{C}_{2}(2)=3$, and $C_{2}(3)=0$. Thus, component 2 is more $\bar{H}$-important than component 1 . It is seen that more $\bar{H}$-important $\Longrightarrow>^{c}$.

Example 2: Let $\phi(\underline{x})=\left[1-\left(1-x_{1}\right)\left(1-x_{2}\right)\right] \cdot\left[1-\left(1-x_{3}\right)\left(1-x_{4}\right)\left(1-x_{5}\right)\right]$.

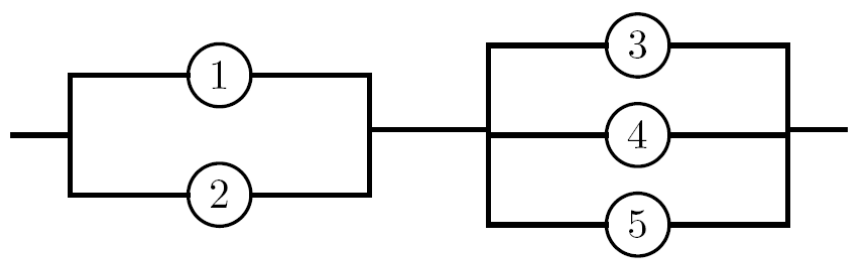

Figure 2: A parallel-series system 
Consider components 1 and 3. Clearly, $\left|C_{1}(1)\right|=\left|C_{3}(1)\right|=0$, and $\left|C_{1}(5)\right|=$ $\left|C_{3}(5)\right|=1 . \quad C_{1}(2)=\{(1,2)\}, C_{1}(3)=\{(1,2,3),(1,2,4),(1,2,5)\} ; C_{1}(4)=$ $\{(1,2,3,4),(1,2,3,5),(1,2,4,5),(1,3,4,5)\} ;\left|C_{3}(2)\right|=0, C_{3}(3)=\{(1,2,3),(3,4$, $5)\}$; and $C_{3}(4)=\{(1,2,3,4),(1,2,3,5),(1,3,4,5),(2,3,4,5)\}$. Thus, $\left|C_{1}(d)\right| \geq$ $\left|C_{3}(d)\right|$ holds for all $1 \leq d \leq 5$, but $1>^{c} 3$ does not hold. That is, more H-important $\Longrightarrow>^{c}$. The fact that $>_{c} \not$ more H-important follows from (II) stated in section 1 and Theorem 3 of Hwang (2001, p.77).

Suppose that $p_{2}=p_{4}=p_{5}$ in Example 2 and two components are to be allotted to node 1 and node 3, one for each node. By Theorem 2.5, the more reliable component should be assigned to node 1 rather than node 3 to achieve better system reliability. The reliability function, $h(\underline{\mathrm{p}})=\left(p_{1}+p_{2}-p_{1} p_{2}\right) \cdot\left(p_{3}+p_{4}+\right.$ $\left.p_{5}-p_{3} p_{4}-p_{4} p_{5}-p_{3} p_{5}+p_{3} p_{4} p_{5}\right)$. Let $p_{1}=0.6, p_{3}=0.8$ and $p_{2}=p_{4}=p_{5}=0.7$ for instance. Then $h(0.6,0.7,0.8,0.7,0.7)=0.86416$, and $h(0.8,0.7,0.6,0.7,0.7)=$ 0.90616 . Suppose that components 2,4 and 5 possess different reliability values, say for instance, $\left(p_{2}, p_{4}, p_{5}\right)=(0.9,0.5,0.5)$. Then $h(0.6,0.9,0.8,0.5,0.5)=0.912$; and $h(0.8,0.9,0.6,0.5,0.5)=0.882$.

\section{References}

Barlow, R. E. and Proschan, F. (1981). Statistical theory of reliability and life testing: probability models, Silver Spring, MD: To begin with. (********* what is it? A book or something? ${ }^{* * * * *}$ )

Boland, P.J., Proschan F., and Tong Y. L. (1989). Optimal arrangement of components via pairwise rearrangements. Naval Research Logistics Quarterly 36, 807-815.

Butler, D. A. (1979). A complete importance ranking for components of binary coherent systems with extensions to multi-state Systems. Naval Research Logistics Quarterly 4, 565-578.

Hwang F. K. (2001). A new index of component importance. Operations Research Letters 28, 75-79.

Hwang F. K. (2005). A Hierarchy of Importance Indices. IEEE Transactions on Reliability 54, 169-172.

Meng, F. C. (1995) Some further results on Ranking the Importance of System Components. Reliability Engineering and System Safety 47, 97-101.

Received October 3, 2007; accepted November 1, 2007.

Fan C. Meng

Institute of Statistical Science

Academia Sinica

Taipei 11529, Taiwan

fcmeng@stat.sinica.edu.tw 